\title{
Sieroprevalenza di infezione da HBV e HCV tra pazienti in dialisi
}

\author{
Rosa Anna Leone, Pasquale Minchella, Salvatore Nisticò, Giuseppe Ivan Potente, \\ Domenico Caruso, Elvira Falvo', Salvatore Cantafio', Carmela Cosentino \\ U.O. di Microbiologia e Virologia, Azienda Sanitaria N. 6, Lamezia Terme (CZ) \\ ' U.O. di Nefrologia e Dialisi, Azienda Sanitaria N. 6, Lamezia Terme (CZ)
}

\section{Seroprevalence of HBV and HCV among dialysis patients}

Key Words: HCV, HCV-RNA; HBV, dialysis

\section{SUMMARY}

The aim of the present study was to investigate the seroprevalence of HBV and HCV among dialysis patients in the Lamezia Terme (CZ) area during the period 1999-2002. Sera from 63 patients in haemodialysis (HD) and I0 patients in peritoneal dialysis (PD) were analyzed with a follow-up every three months for $\mathrm{HBs} A g, \mathrm{HBc} A b$, $\mathrm{HBsAb}$, anti-HCV and anti-HIV (Elisa Test,AxSYM, Abbott); we analyzed reactive sera for anti-HCV by using supplemental test (RIBA Test, Ortho); we also looked for viremia (RT-PCR Amplicor, Roche Diagnostics) and HCV genotypes (Inno-Lipa HCV II, Innogenetics). The results show that, among the HD patients, 3 were HBsAg positive (Chronic Infection) and $7 \mathrm{HBcAb}$ and $\mathrm{HBsAb}$ positive/HBsAg negative (Passed Infection); 14 individuals were anti-HCV positive. No patients in PD were positive for $\mathrm{HBV}$ and $\mathrm{HCV}$ markers. The prevalence of chronic HBV infection was $4.8 \%$ (instead of $3 \%$ in other Dialysis Units), that of anti-HCV positive was $22 \%$ (in others $24 \%$ $33 \%$ ); among anti-HCV positive patients, the HCV-RNA prevalence was $79 \%$ (instead of $80 \%$ ); the most recurrent $\mathrm{HCV}$ genotype was $2 \mathrm{a} / 2 \mathrm{c}$ (instead of $\mathrm{Ib}$ in general population). These findings lead us to hypothesize that the environmental transmission in the dialysis setting is tightly correlated to the risk of HBV and HCV infection.

\section{INTRODUZIONE}

I pazienti con insufficienza renale cronica sottoposti a dialisi sono esposti ad un alto rischio di infezioni virali trasmissibili per via parenterale, soprattutto infezioni da virus dell'Epatite B (HBV) e dell'Epatite C (HCV).

Il trattamento emodialitico richiede un accesso vascolare per periodi prolungati in un ambiente dove contemporaneamente sono presenti più pazienti; esistono pertanto più possibilità di trasmissione di agenti infettivi, diretta o indiretta attraverso materiale contaminato, superfici ambientali o mani del personale ospedaliero. I pazienti in dialisi sono generalmente immunodepressi e tale condizione aumenta la loro suscettibilità alle infezioni; richiedono inoltre frequenti ospedalizzazioni per patologie spesso associate ed hanno così un ulteriore rischio di esposizione (3).

La possibilità di trasmissione dell'infezione da HBV nei Centri Dialisi si è ridotta notevolmente, soprattutto con l'introduzione di misure preventive idonee, come la vaccinazione anti-Epatite $B$ dei soggetti non immuni ed il trattamento dialitico in ambiente separato dei pazienti con epatite B cronica (HBsAg positivi) (13). Nell'ultimo decennio infatti la prevalenza di infezione cronica da HBV si è ridotta nella popolazione generale dal 3\% al 1.2\%-2\% e nei Centri Dialisi addirittu- ra dal $12 \%$ al $3 \%(4,10,17)$.

I pazienti in dialisi rappresentano tuttavia un gruppo ad alto rischio per quanto riguarda l'infezione da HCV: in Italia la prevalenza media di soggetti con anticorpi anti-HCV nella popolazione generale è variabile dal $3.2 \%$ al nord fino al $12.6 \%$ al sud; la prevalenza di pazienti in dialisi con anticorpi anti-HCV risulta invece nettamente più elevata, variando dal $24 \%$ al $33 \%$ nei vari Centri $(7,16)$. Non essendo ancora disponibile un vaccino anti-Epatite $\mathrm{C}$, la prevenzione dell'infezione HCV deve basarsi soprattutto sulla scrupolosa applicazione delle "precauzioni standard" e delle "precauzioni speciali per i Centri Dialisi", nonché sull'utilizzo di monitors "dedicati" ai pazienti anti-HCV positivi $(2,3)$.

\section{SCOPO DEL LAVORO}

Scopo di questo lavoro è valutare la prevalenza dei marcatori di infezione virale da HBV, $\mathrm{HCV}$ e HIV-1/2 nei pazienti sottoposti a dialisi negli anni 1999-2002 presso l'U.O. di Nefrologia e Dialisi e monitorati presso l'U.O. di Microbiologia e Virologia dell'Azienda Sanitaria n. 6 di Lamezia Terme (CZ).

\section{MATERIALI E METODI}

Sono stati presi in considerazione 63 pazienti in emodialisi (HD) e 10 pazienti in dialisi peritonea- 
le (PD) prevalentemente domiciliare. I soggetti in $\mathrm{HD}, 32$ maschi e 31 femmine con età media di circa 61 anni, avevano un tempo medio di dialisi di circa 7 anni; i soggetti in PD, 7 maschi e 3 femmine con età media di circa 62 anni, avevano un tempo medio di dialisi di circa 3 anni. I pazienti, controllati mensilmente per gli indici di citolisi epatica, sono stati monitorati a cadenza trimestrale, invece che semestrale come generalmente raccomandato, con un test di screening immunoenzimatico per i marcatori sierologici di infezione da $\mathrm{HBV}$, HCV ed HIV; tale procedura, applicata quando non c'erano indicazioni per anticipare il controllo, è stata seguita tenendo conto del tempo medio di sieroconversione anti-HCV nel paziente in dialisi, maggiore che nel resto della popolazione. Per quei pazienti in cui il test di screening non è stato sufficiente per la definizione dello stato sierologico anti-HCV, sono stati eseguiti esami aggiuntivi, sia il test di conferma (spesso non conclusivo nei soggetti immunodepressi come quelli in trattamento dialitico), sia la ricerca della viremia (HCV-RNA), unico marker certo di infettività; nei pazienti risultati viremici è stato poi determinato il genotipo $\mathrm{HCV}$.

CAMPIONI: Il siero è stato separato mediante centrifugazione $(2.500 \mathrm{~g}$ per $10 \mathrm{~min})$ entro 2 ore dal prelievo per i tests di screening e di conferma, mentre per la ricerca dell'HCV-RNA il plasma è stato separato mediante centrifugazione $(1.500 \mathrm{~g}$ per 20 minuti) entro 1 ora dal prelievo. I campioni non analizzati in giornata sono stati aliquotati rispettivamente a $-20^{\circ} \mathrm{C}$ ed a $-80^{\circ} \mathrm{C}$.

METODI: Test di screening: per la ricerca di HBsAg, HBcAb totali, HBsAb ed anti-HIV-1/2 è stato utilizzato un metodo immunoenzimatico a cattura di microparticelle (MEIA) su analizzatore AxSYM (Abbott), secondo metodica. Per il test anti-HCV è stato utilizzato il dosaggio AxSYM $\mathrm{HCV}$ versione 3.0, che utilizza 4 antigeni ricombinanti. I risultati, di tipo qualitativo, sono espressi come rapporto $(\mathrm{S} / \mathrm{CO})$ tra il valore del segnale generato dal campione (S) ed il valore limite (CO) che l'analizzatore AxSYM calcola moltiplicando per 0.12 il valore medio del calibratore indice. Per l'interpretazione degli indici S/CO abbiamo adottato una "scala graduata" invece dell'interpretazione indicata in metodica: "Non Reattivo" se l'indice $\mathrm{S} / \mathrm{CO}<0.80$; "Valore Limite" se $\mathrm{S} / \mathrm{CO}$ $>/=0.80<1.20$; "Reattivo Basso" se $\mathrm{S} / \mathrm{CO}>/=$ $1.20<5.00$; "Reattivo" se $\mathrm{S} / \mathrm{CO}>/=5.00$.

Test sierologico di conferma: è stato utilizzato il test "RIBA HCV 3.0 SIA" (Chiron Corporation, distribuito da Ortho Diagnostic Systems), un dosaggio immunoblot qualitativo su strip (SIA) che utilizza antigeni ricombinanti e peptidi sintetici, immobilizzati sotto forma di bande individuali sulle strips. Interpretazione dei risultati: "Negativo" quando nessuna banda è presente; "Indeterminato" quando è presente una sola banda; "Positivo" quando sono presenti almeno due bande.

Ricerca qualitativa HCV-RNA: è stato utilizzato il kit Cobas AMPLICOR HCV test, versione 2 (Roche Diagnostics), che si basa sull'amplificazione polimerasica a catena dopo retrotrascrizione (RT-PCR) di una porzione di 244 basi della regione 5' UTR del genoma di HCV, usando i primers KY80 e KY78-biotinilato. Il kit utilizza per la rivelazione la tecnica di ibridazione su fase solida: l'amplificato è marcato con biotina e si lega a sonde di cattura specifiche adese a particelle magnetiche. La rivelazione avviene con un sistema indiretto tramite avidina coniugata con l'enzima perossidasi (coniugato) ed aggiunta di idoneo substrato (TMB). La sensibilità del kit è di $50 \mathrm{UI} / \mathrm{ml}$.

Quantizzazione HCV-RNA: è stato utilizzato il kit Cobas AMPLICOR HCV Monitor (Roche Diagnostics): lo standard interno di quantizzazione (QS), aggiunto a titolo noto ad ogni estrazione, viene co-estratto ed amplificato simultaneamente al target HCV-RNA mediante gli stessi primers, ma viene rivelato da sonde di ibridazione diverse da quelle dell'amplificato target. La concentrazione di HCV-RNA di ogni campione viene determinata confrontando l'assorbanza del campione amplificato con quella ottenuta dalla rivelazione del QS, standardizzato contro lo standard internazionale $\mathrm{HCV}$ dell'OMS in UI/ml. La sensibilità del kit è di $600 \mathrm{UI} / \mathrm{ml}$.

Genotipizzazione: è stato utilizzato il test "INNO LIPA HCV II" (Innogenetics, distribuito da Bayer), che consiste in una ibridazione inversa su fase solida del prodotto di amplificazione ottenuto con metodo RT-PCR per la ricerca qualitativa dell'HCV-RNA, con sonde specifiche per il riconoscimento dei 6 principali genotipi del virus dell'Epatite $\mathrm{C}(\mathrm{HCV})$ e dei loro più comuni sottotipi, secondo la classificazione di Simmonds. Il genotipo è stato dedotto utilizzando la carta d'interpretazione del kit.

\section{RISULTATI}

I risultati ottenuti nei 63 pazienti in HD, per quanto riguarda la presenza dei marcatori sierologici di infezione da HBV, HCV ed HIV sono di seguito indicati.

- Infezione da HBV: sono state osservate n. 3 $(4.8 \%)$ reattività per $\mathrm{HBsAg}$ e $\mathrm{HBcAb}$ con $\mathrm{HBsAb}$ assenti, da interpretare come infezione cronica e n. 7 (11.1\%) reattività per $\mathrm{HBcAb}$, con $\mathrm{HBsAg}$ negativo e presenza di titolo superiore a 
$10 \mathrm{mUI} / \mathrm{ml}$ di HBsAb, da interpretare come infezione pregressa;

- Infezione da HCV: al test di screening anti-HCV si sono avuti n. $14(22.2 \%)$ risultati che hanno richiesto, per l'interpretazione finale dello stato sierologico, esami supplementari (test RIBA e ricerca HCV-RNA);

- Coinfezione HBV-HCV: soltanto n. 2 (3.2\%) dei 3 pazienti con reattività $\mathrm{HBsAg}$ presentavano contemporanea reattività anti-HCV, confermata con i test supplementari (tabella 1).

\begin{tabular}{ccccc}
\hline N. PZ (63) & HbsAg & HbsAb & HbcAb & HCV \\
\hline I & + & - & + & - \\
\hline 2 & + & - & + & + \\
\hline 7 & - & + & + & - \\
\hline I2 & - & + & - & + \\
\hline $4 \mathrm{I}$ & - & + & - & - \\
\hline
\end{tabular}

Tabella I. Marcatori sierologici Epatite $B$ ed Epatite $C$ nei pazienti in emodialisi

La ricerca dell'HCV-RNA mediante PCR, nei 14 pazienti per i quali si è dovuto procedere ad un approfondimento diagnostico, ha dato i seguenti risultati: n. $11(79 \%)$ positivi e n. $3(21 \%)$ negativi (figura I). Su tali 3 pazienti non viremici il test RIBA ha dato risultato positivo, permettendo di escludere una falsa positività al test di screening. Lo stato sierologico di questi 14 pazienti è stato infine interpretato come di positività anti-HCV, visto il risultato positivo di almeno uno dei due test supplementari (1). Negli 11 pazienti viremici è stata eseguita la quantizzazione dell'HCV-RNA che, in $10(91 \%)$, ha dato un risultato maggiore di $500.000 \mathrm{UI} / \mathrm{ml}$; la determinazione del genotipo $\mathrm{HCV}$ ha dato i seguenti risultati: $2 \mathrm{a} / 2 \mathrm{c}$ n. 6 (54.5\%), 1b n. 4 (36.4\%), 3a n. 1 (9.1\%).

Figura I. Risultati ricerca HCV-RNA mediante $P C R$ nei 14 pazienti anti-HCV positivi in emodialisi

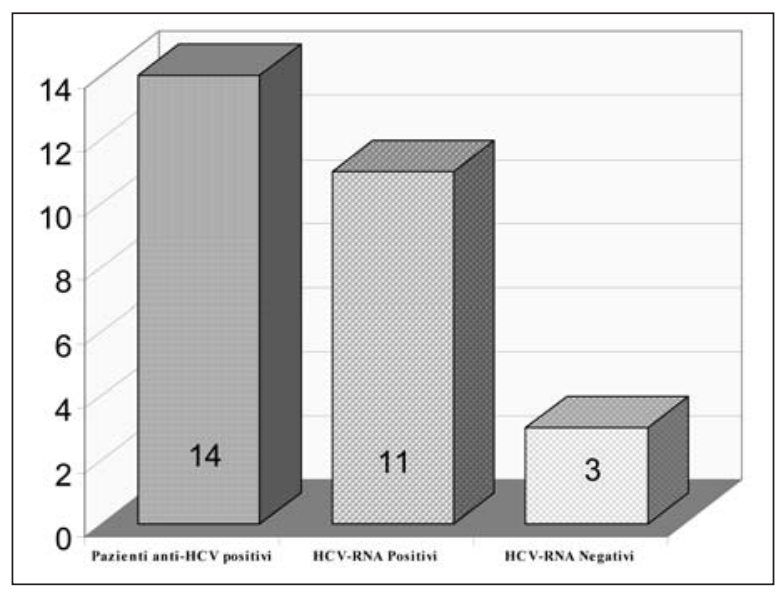

Tra i 14 pazienti anti-HCV positivi è stata effettuata un'indagine retrospettiva, basata sulla storia anamnestica e sui dati di laboratorio annotati nelle cartelle cliniche: è risultato che, prima dell'inizio della HD, n. 5 (35.7\%) pazienti erano già "antiHCV reattivi", mentre n. 9 (64.3\%) erano "antiHCV non reattivi"; n. 5 sieroconversioni antiHCV sono comparse durante i primi 3 anni di dialisi, mentre n. 4 dopo più di 10 anni di trattamento dialitico. Si è notato che, tra i 5 pazienti "antiHCV reattivi" già prima della HD, n. 4 avevano ricevuto più trasfusioni precedentemente al trattamento dialitico.

Durante il periodo di osservazione dello stato sierologico dei 63 pazienti in HD sono state individuate e monitorate n. 3 sieroconversioni anti$\mathrm{HCV}$ in pazienti senza pregresse emotrasfusioni o altri fattori di rischio extra-dialitico. Le sieroconversioni sono state messe in evidenza tempestivamente, valutando con attenzione il test di screening immunoenzimatico e considerando un indice $\mathrm{S} / \mathrm{CO}>/=0.80$ come un segnale d'allarme, che ha indotto a monitorare i pazienti sierologicamente. Le sieroconversioni, documentate da un progressivo aumento dell'indice, sono state anche confermate dalla presenza di viremia, rivelata precocemente e rimasta persistentemente positiva. In un solo paziente, che ha mantenuto costantemente un titolo HCV-RNA $<600 \mathrm{UI} / \mathrm{ml}$, dopo circa un anno si è avuta negativizzazione della viremia, confermata da ripetuti controlli. Il RIBA, eseguito tempestivamente sui 3 pazienti, ha dato 2 risultati Indeterminati (presenza della sola banda c22) ed 1 risultato Positivo; a distanza di un anno il test è stato ripetuto nei due pazienti con risultato Indeterminato: si è osservata positivizzazione del test in un paziente, mentre nell'altro il risultato è rimasto Indeterminato, pur in presenza di positività del test HCV-RNA.

Nei 10 pazienti in PD non è stato rivelato alcun marcatore di infezione per HBV e HCV. Nessun paziente (sia in $\mathrm{HD}$ che in $\mathrm{PD}$ ) è risultato reattivo per anti-HIV-1/2.

\section{DISCUSSIONE E CONCLUSIONI}

Dall'analisi dei dati relativi ai pazienti in HD è risultata una prevalenza di infezione cronica da HBV del 4.8\% (3/63), lievemente superiore rispetto alla media nazionale attuale del $3 \%(9,11,13)$. I 3 pazienti con infezione cronica erano sottoposti a dialisi da circa 20 anni; sono diventati HBsAg positivi nei primi anni di trattamento e quindi precedentemente all'introduzione della vaccinazione anti-Epatite B. La prevalenza di anticorpi anti$\mathrm{HCV}$ nel campione analizzato è risultata del $22.2 \%$ $(14 / 63)$, lievemente inferiore ai dati ottenuti (tra $24 \%$ e $33 \%$ ) nei Centri Dialisi che hanno partecipato ad una indagine epidemiologica nazionale sull'infezione da HCV (tabella 2) $(14,16,18)$. 


\begin{tabular}{lccc}
\hline & $\begin{array}{c}\text { PREVALENZA } \\
\text { AREA } \\
\text { LAMETINA }\end{array}$ & $\begin{array}{c}\text { PREVALENZA } \\
\text { ALTRI } \\
\text { CENTRI }\end{array}$ & $\begin{array}{c}\text { PREVALENZA } \\
\text { POPOLAZIONE } \\
\text { GENERALE }\end{array}$ \\
\hline $\begin{array}{l}\text { Infezione } \\
\text { cronica HBV }\end{array}$ & $\begin{array}{c}(3 / 63) \\
\text { Anticorpi anti-HCV }\end{array}$ & $3 \%$ & $1.2 \%-2 \%$ \\
& $22 \%$ & $24 \%-33 \%$ & $3.2 \%-12.6 \%$ \\
& $(14 / 63)$ & & \\
\hline
\end{tabular}

Tabella 2. Prevalenza di infezione cronica HBV e di anticorpi anti-HCV nei pazienti in emodialisi e nella popolazione generale

La prevalenza di viremia tra i pazienti anti-HCV positivi è risultata del $79 \%$, in accordo quindi alla prevalenza dell' $80 \%$ rilevata negli altri Centri Dialisi (8).

Il genotipo $\mathrm{HCV}$ più diffuso è risultato essere il $2 \mathrm{a} / 2 \mathrm{c}$, seguito dall' $1 \mathrm{~b}$ e dal $3 \mathrm{a}$, come indicato in altri lavori sui pazienti in dialisi, mentre nella popolazione generale il genotipo più diffuso è l'1b (7).

L'attenta valutazione dell'indice $\mathrm{S} / \mathrm{CO}$ del test di screening anti-HCV, soprattutto nei soggetti immunodepressi ed a rischio di infezione quali sono i pazienti in dialisi, ci ha permesso di eseguire tempestivamente $i$ test diagnostici supplementari e, nello stesso tempo, allertare il personale del Centro Dialisi, per monitorare i pazienti con indagini più approfondite.

Durante il periodo di osservazione dei 10 pazienti in PD non è stato rivelato alcun marcatore sierologico di infezione $\mathrm{HBV}$ ed $\mathrm{HCV}$ : questi dati devono però ritenersi ancora preliminari, visto il campione numericamente limitato e la durata, ancora breve, di questo tipo di trattamento; comunque, sia la diversità delle modalità di dialisi, sia l'ambiente domiciliare in cui viene prevalentemente eseguito, espongono i pazienti ad un rischio indubbiamente minore.

Le caratteristiche del virus dell'epatite B, soprattutto la sua resistenza all'ambiente esterno ed ai comuni disinfettanti, hanno reso consolidata la pratica dell'isolamento dei pazienti HBsAg positivi. Tale misura non appare invece necessaria per i pazienti anti-HCV positivi perché il virus HCV possiede caratteristiche meno aggressive, anche se va incontro a continue mutazioni, causa di persistenza dell'infezione; è comunque raccomandato l'uso di monitor "dedicati" ai pazienti antiHCV positivi $(2,3)$.

L'infezione HCV nei Centri Dialisi non può dirsi ancora sotto controllo, nonostante una riduzione della prevalenza registrata in questi ultimi anni: si segnalano ancora sieroconversioni in pazienti senza anamnesi emotrasfusionale o altri fattori di rischio extra-dialitico, quindi sieroconversioni da probabile trasmissione nosocomiale di tipo parenterale inapparente $(4,6,15)$. L'ambiente dialitico è una realtà particolare, con proprie specifiche peculiarità epidemiologiche come l'elevata prevalenza di pazienti anti-HCV positivi, 1' $80 \%$ dei quali è persistentemente viremico e quindi infettante. È ampiamente riconosciuto che nei Centri Dialisi sono necessarie precauzioni aggiuntive, più articolate e severe delle normali precauzioni standard: la loro scrupolosa applicazione è fondamentale per evitare nuove sieroconversioni da contaminazione ambientale $(5,12)$.

Il problema del rischio di trasmissione delle infezioni da HBV ed HCV nei Centri Dialisi risulta ancora oggi di grande rilevanza epidemiologica: obiettivi primari da raggiungere, oltre che rendere più efficace e completo il programma di prevenzione immunologica, sono soprattutto una maggiore sensibilizzazione del personale ospedaliero ed una sempre più attenta applicazione delle precauzioni per la prevenzione della diffusione nosocomiale delle infezioni. Dalla nostra esperienza è risultato che il microbiologo può avere un ruolo sempre più attivo nell'ambito della sorveglianza delle infezioni ospedaliere, segnalando tempestivamente le nuove sieroconversioni, orientando in modo mirato la scelta del tipo di indagini di approfondimento da eseguire, affiancando il clinico nella interpretazione diagnostica.

\section{BIBLIOGRAFIA}

1. CDC. Guidelines for laboratory testing and result reporting of antibody to hepatitis $\mathrm{C}$ virus. MMWR 2003; 52 (RR-3): 1-15.

2. CDC. MMWR Update: universal precautions for prevention of transmission of HIV, HBV, and others blood born pathogens in health-care setting. JAMA 1988; 260: 462-5.

3. CDC. Recommendations for preventing transmission of infections among chronic hemodialysis patients. MMWR 2001; 50 (RR-5): 1-45.

4. Cendoroglo Neto M, Draibe SA, Silva AE, et al. Incidence of and risk factors for hepatitis B virus and hepatitis $\mathrm{C}$ virus infection among haemodialysis and CAPD patients: evidence for environmental transmission. Nephrol Dial Transplant 1995; 10 (2): 240-6.

5. Franco E, Olivadese A, Valeri M, Albertoni F, Petrosillo N. Control of hepatitis B virus infection in dialysis units in Lazium, Italy. Nephron 1992; 61 (3): 329-30.

6. Irish DN, Blake C, Christophers J, et al. Identification of hepatitis $\mathrm{C}$ virus seroconversion resulting from nosocomial transmission on a haemodialysis unit: implications for infection control and laboratory screening. J Med Virol 1999; 59 (2): 135-40.

7. Lombardi M, Cerrai T, Geatti S, et al. Risultati dell'indagine epidemiologica nazionale sull'infezione da HCV nei pazienti in dialisi, curata dalla filiale italiana dell'EDTNA/ERCA. In Atti del XVII Congresso Nazionale EDTNA/ERCA, Obiettivo qualità nel nursing nefrologico: processi, strumenti, risultati, Bari. 1998: 59-70.

8. Lombardi M, Dattolo P, Pizzarelli F, et al. Prevalenza e significato clinico della viremia $\mathrm{C}$ nei pazienti in 
emodialisi. Giorn It Nefrol 1994; 11: 271-7.

9. Lopez-Alcorocho JM, Barril G, Ortiz-Movilla N, et al. Prevalence of hepatitis $\mathrm{B}$, hepatitis $\mathrm{C}$, GB virus $\mathrm{C} /$ hepatitis $\mathrm{G}$ and TT viruses in predialysis and hemodialysis patients. J Med Virol 2001; 63 (2): 103-7.

10. Mele A, Spada E. Epidemiologia delle infezioni acute da virus epatitici a trasmissione parenterale. Istisan 2000; 32: 141-7.

11. Mioli VA, Balestra E, Bibiano L, et al. Epidemiology of viral hepatitis in dialysis centers: a national survey. Nephron 1992; 61 (3): 278-83.

12. Petrosillo N, Gilli P, Ippolito G, et al. Raccomandazioni per il controllo dell'infezione da HCV in dialisi. Giorn It Nefrol 1995; 12: 291-8.

13. Petrosillo N, Puro V, Ippolito G. Prevalence of human immunodeficiency virus, hepatitis B virus and hepatitis $\mathrm{C}$ virus among dialysis patients. The Italian multicentric study on nosocomial and occupational risk of blood-borne infections in dialysis. Nephron 1993; 64 (4): 636-9.

14. Rivanera D, Lilli D, Lorin G. et al. Detection of antibodies to hepatitis $\mathrm{C}$ virus in dialysis patients. Eur $\mathrm{J}$ Epidemiol 1993; 9 (1): 55-8.

15. Sampietro M, Badalamenti S, Graziani G. Nosocomial hepatitis $\mathrm{C}$ in dialysis units. Nephron 1996; 74: 25160.

16. Scotto G, Avcella F, Panunzio M, et al. Hepatitis C virus infection in four haemodialysis units of southern Italy: epidemiological report. Eur J Epidemiol 1999; 15 (3): 217-23.

17. Stroffolini T, Guadagnino V, Chionne P, et al. A population based survey of hepatitis $B$ virus infection in a southern Italian town. Ital J Gastroenterol Hepatol 1997; 29 (5): 415-8.

18. al-Wakeel J, Malik GH, al-Mohaya S, et al. Liver disease in dialysis patients with antibodies to hepatitis C virus. Nephrol Dial Transplant 1996; 11 (11): 2262-8.

\section{Rosa Anna Leone}

Unità Operativa di Microbiologia e Virologia

Via A. Perugini

88046 Lamezia Terme (CZ)

Tel.: 0968 208761; Fax: 0968463113

E-mail: minchellap@libero.it 